\title{
Parking Management System Optimization on the Street In Order To Reduce Transport Problems By Using A Prototype Of Integrated Parking Management System (Case Study : Parking Area at Alun-Alun Utara Kota Pekalongan)
}

\author{
Sahid Bismantoko, Asep Haryono and Tri Widodo
}

\begin{abstract}
These the problem of parking systems on the streets is a classic problem that occurs from year to year, many solutions are offered in solving parking problems on the street. The problem is not only related to congestion due to exit and enter the vehicle from the parking lot but also the problem of parking management becomes the current polemic. Optimization of integrated parking management system tries to provide solution in parking management that is by giving real time vehicle parking data to server so that manager can access data then make report or policy related parking. This system can be implemented multi-parking area in an integrated manner. In addition, this system can also provide vehicle parking monitoring for security vehicles in the parking lot. The development of this system for parking vehicle identification is supported by smart camera. In the prototype, the system test is carried out in day and night conditions, to anticipate the difference of pixel intensity on the vehicle object then in developing vehicle detection program using adaptive thresholding method. The results show that the identification vehicle program and sending data in real time from smart camera to server have been running quite well during the day and night.
\end{abstract}

Index Terms-Optimization Parking Management, Information System, Monitoring System

\section{INTRODUCTION}

$\mathrm{T}$ HE complexity of transportation problems in urban areas especially land transportation such as traffic congestion, public transportation, polution, undisciplined in parking of vehicles on the street and so on. One of the problems is parking on the street where many offenses are done such as

Sahid Bismantoko is with Agency for The Assessment and Application of Technology - Indonesia (e-mail: sahid.bismantoko@bppt.go.id).

Asep Haryono is with Agency for The Assessment and Application of Technology - Indonesia.

Tri Widodo is with Agency for The Assessment and Application of Technology - Indonesia vehicle parking is not on the parking area, and parking vehicle that does not follow the parking marker so it is inefficiency of parking space. In addition, management issues in vehicle parking are a difficult part to control, as this requires continous upervision. Currently there are several e-parking systems that have been installed in several locations, especially large cities in Indonesia, as well as eparking models that use smart cards. In this research one of the alternative technologies offered in the parking management system is the integration of information systems with smart cameras. Integrated parking management system is expected to provide convinience for users in this case the local government and public. This integrated parking management system has the benefit of providing real-time parking data, in addition to monitoring vehicle parking as part of vehicle parking security. Technically this system can be integrated between other parking areas so that local governments can monitor the condition of every parking area.

\section{RELATED WORK}

Smart parking researches have been conducted which have the purpose of providing convenience for parking users by utilizing mobile applications [2] [3] [6] [9] [10], in addition to providing convenience and shortening time in finding parking spaces in the area parking [6] [8]. The parking management system developed using image processing-based cameras [1] [3], [8], [9], also used sensors for the detection of vehicles in and out of parking areas [9]. While the object identification as the target is the vehicle body [8] [9] or the vehicle number plate [2] [4] [5].

An integrated parking management system is the networking for many parking areas with the Internet of Thing (IoT) [7] has been researched. The study of parking management systems that have been conducted using different approaches, such as the development of smart parking systems by using a combination of image processing and sensor for vehicle detection and available parking spaces [9] in which the paper aims to develop intelligent 
transportation systems in managing and controlling these services, developing mobile devices to provide information regarding available parking lots, developing inexpensive hardware related to parking systems [9].

\section{The CONCEPT OF INTEGRATED PARKING MANAGEMENT SYSTEM}

Development of Prototype of Integrated Parking Management System is focused on the development of information and communication technology for parking system applications supported by smart cameras to obtain vehicle parking data in real time and monitoring in order to improve the security of parking vehicles.

Architectural prototype of Integrated Parking Management System as in Fig. 1, where the process of capturing vehicle objects in the parking area is done through the smart camera then the vehicle is verified whether the correct vehicle will be parked or not before the data is sent to the server as parking vehicle data.

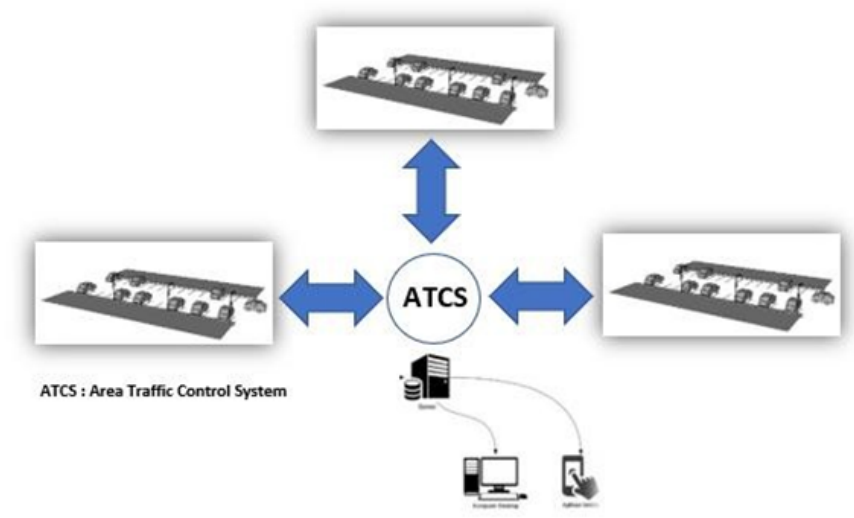

Fig. 1. Concept of Integrated Parking Management System on the street

In this concept the development stage as shown (1) is as follows:

1) The object captured by the camera will be processed whether the vehicle is parked or not

2) Data from the smart camera will be sent to the server

3) Data can be displayed in a web-based application program for the needs of the relevant agencies

4) Data may be displayed in mobile application programs as information to public.

\section{Development of InTEgrated Parking MANAGEMENT SYSTEM}

Detailed description of the integrated parking management system in Fig. 2, as shown below:

1) The camera will capture the parking vehicle as data which is sent to the server periodically.

2) As users of desktop computers, Web pages can be accessed by entering URL addresses while mobile application users access android applications that have
Web View Client installed.

3) Web Server Software (apache 2.0) will recognize requests from a PHP script file and then the server will translate the file with the PHP plug-in before being responded by the request page

4) PHP with the Yii Framework will connect to the MySQL database and request content that matches the web page.

5) The MySQL database will respond by sending the content requested by the PHP script.

6) The PHP script stores the content into one or more PHP variables and then an echo function will output the appropriate content on the web page.

7) The PHP plugin makes a copy of HTML created by the Web server.

8) Web server sends an HTML to Web browser / Web view written with HTML file provided by PHP plug-in.

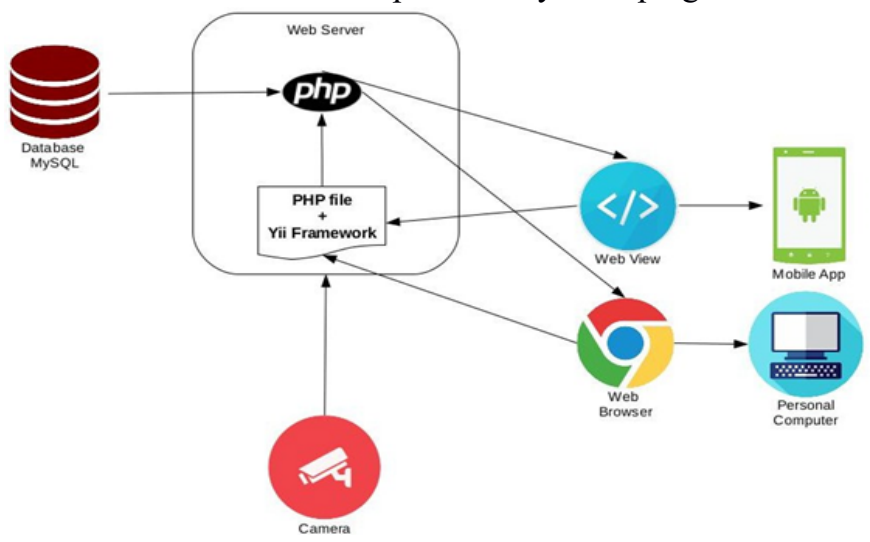

Fig. 2. Integrated Parking Management System

\section{A. Hardware and Software}

Specifications Software used:

1) Linux as Operating System.

2) PHP 5 as a programming language.

3) Yii 1.1.11 as a PHP Framework.

4) MySQL database as Database Management System.

5) MySQL Workbench for database design and modeling.

6) Netbeans IDE 8.1 as Integrated Development Environment.

7) Libreoffice Math 5.1.6.2 for the creation of detailed flowcharts.

8) Google maps API as Map View to display the map.

9) Mozilla Firefox and Google Chrome.

10) Android Studio 2.3 As an android IDE.

Hardware used: Mini PC, POE, IP Camera, Modem, Outdoor Box and Terminal Boxes, Power Cable and UTP Cable

\section{B. Smart Camera Program Development}

The development of smart cameras for the identification of vehicle- based image processing has done a lot of research, in this research that distinguishes with other research are:

1) The program is developed to work in day and night 
conditions, so that the pixel intensity difference is attempted to be adapted by using adaptive thresholding.

2) Verification method to determine whether the vehicle is properly parked or just "pickup or get off", so it does not enter as data in the count as a parking vehicle.

The software used in this development are as follows $\mathrm{C} \mathrm{++}$ main program and Open CV.

\section{Integrated Information System Development Method}

The method used in the development of information systems is the Rapid Application Development (RAD) method. RAD is the incorporation of several structured methods or techniques. This method uses prototyping methods and other structured techniques to determine user requirements and information system design. The development process includes:

1) Learn whether the system development project meets the criteria.

2) Make a model of the priority functions.

3) Choose which prototype is reviewed.

4) Implementation of information systems.

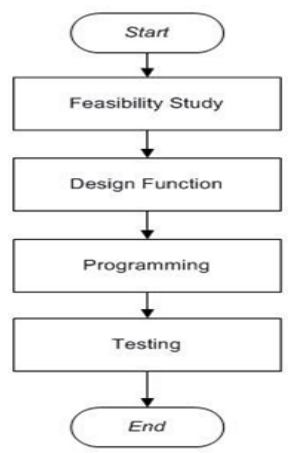

Fig. 3. Integrated Information System Development Method

From Fig. 3 above the description of each stage is as follows:

I. Feasibility Study

At this stage there are several steps that need to be prepared that is:

1) Problem analysis

The problem of real time data becomes important in the optimization of parking management where verification is required to compare between real time data suported by smart camera and data manually other the issue of vehicle security parked on the street of something of concern.

\section{2) Needs analysis}

Needs analysis is divided into two namely the functional needs and non-functional needs. Functional needs are the needs associated with processing and data transformation, while non-functional requirements are the needs associated with the needs of hardware and software.

3) System analysis

Requirement of making integrated information system software for application of parking management optimization on street.

\section{Design Function}

Designing detailed system functions includes:

\section{1) Process Design}

The description of a system to be made is called the process design. The design process consists of several context diagrams. Based on the results of the analysis, the system will be designed to provide final information in the form of details of a system.

\section{2) Database Design}

At this stage is divided into 3 stages of conceptual design, design logic and physical design. The 3 stages is input design, output design and interface design. The input design is designed for camera input data. The design of output in the form of real data sent to the server and then processed as data to be received in the interface as a view and interface design is required to make it easier for users to use the system.

\section{3) Programming}

- Coding

This stage realize the design functions that have been made include:

$>$ Process Implementation

$>$ Database Implementation

$>$ Input Implementation

>Implementation Output

$>$ Interface Implementation

- Fixed minor bugs on the system

At the coding stage will find some bugs that need to be fixed. This stage refines a coding.

4. Testing

- System Integration and Testing

This stage is done to determine the performance of system functions.

- Evaluation and Recommendation

Evaluation of software, which can be further developed.

III. Framework

Framework as a collection of scripts (especially class and function) that can handle various problems in programming such as connection to database, calling variables, files, in addition to it to be faster in building applications.

Yii Framework implements a model-view-controller (MVC) design pattern that is widely adopted in web programming. MVC aims to separate the business logic, the user in order to more easily change each part without affecting the others. 


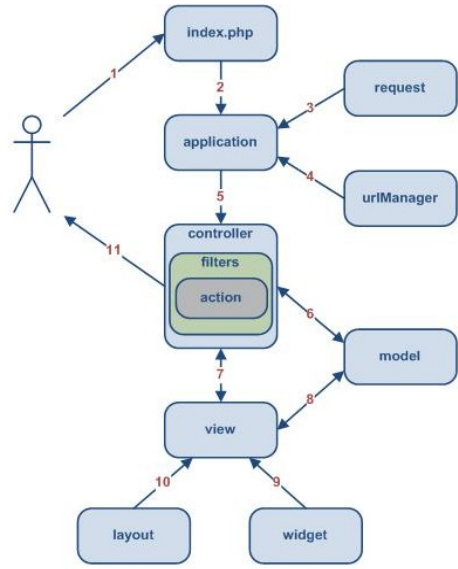

Fig. 4. Yii Framework Workflow

In general the workflow is as follows (in Fig. 4):

1) The user creates a request with the URL and the Web server handles the request by running the bootstrap index.php script.

2) The bootstrap script creates an application instance and runs it.

3) Applications get details of user request information from an application component named request.

4) The application specifies the controller and action requested with the help of an application component named urlManager. In this example, the controller is a post that refers to the PostController class; and action is a show whose true meaning is determined by the controller.

5) The application creates the requested controller instance to further handle user requests. The controller determines the show action refers to a method named actionShow in the controller class. Then create and run filters (eg access control, measurement) related to this action. Action executed if allowed by filter.

a. Read Post model where ID is 1 from database.

b. Prepares a view named show with Postmodel.

c. View reads and displays Post model attributes.

d. View runs multiple widgets.

e. View prepares paired results in layout.

f. Action ends the view creation and displays the end result to the user.

\section{$I V$. Information System Database}

Databases are necessary to store data as storage. The design of the database should be made so that the data stored in accordance with the needs and when accessing is not heavy. There is a relationship between one table to another to set the database. Here is the database table in Fig.5 relation of vehicle parking management system.

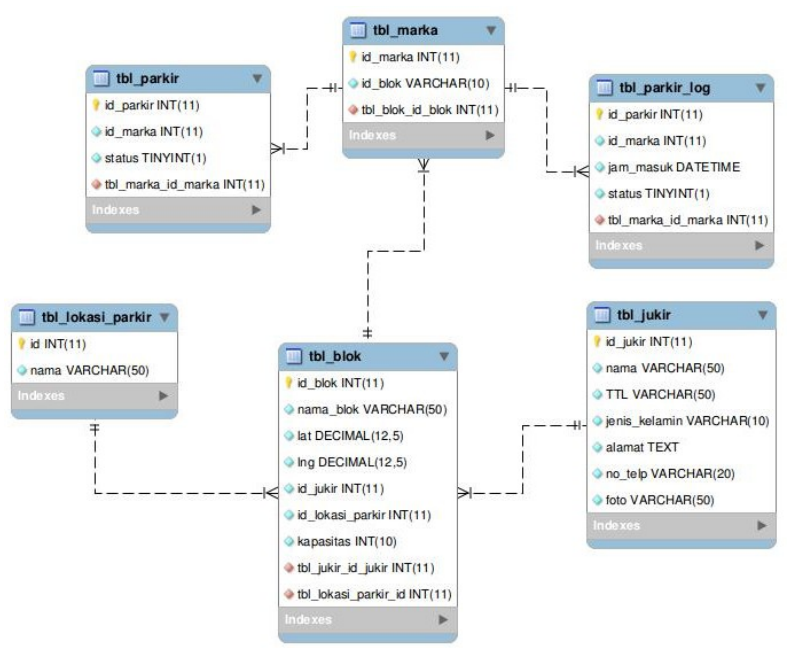

Fig. 5. Information System Database

\section{TESTING}

Prototype of integrated parking management system Testing implemented in the parking area. The testing is planned to be performed using number of installed cameras, mini PCs, modems and Power Over Ethernet (POE) of 2 units each, and testing are conducted day and night.

In this testing aims to determine the effectiveness of the use of smart cameras in identifying parking vehicles, as well as testing the delivery of real data of vehicle identification to the server.

The test was done with two cameras, while the test results as follows:

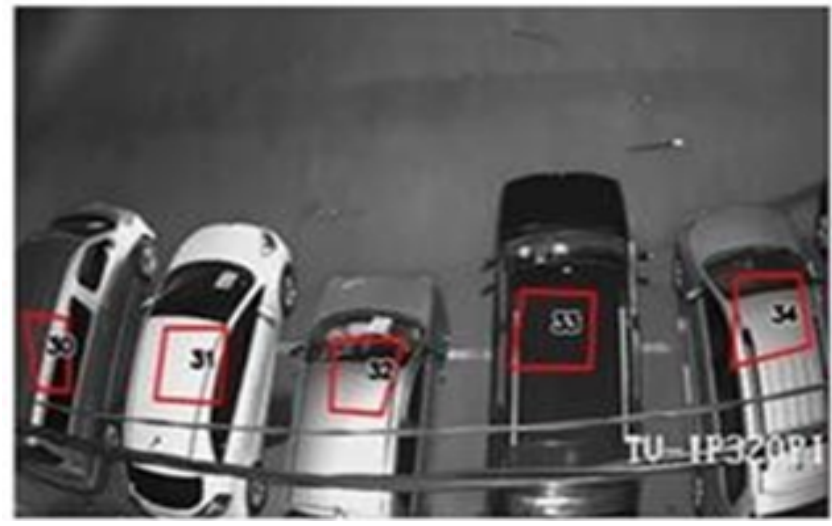

Fig. 6. Vehicle Parking Identification Results Using Smart Cameras At Night From the above test results as shown in Fig. 6, the test runs at night, the vehicle identification program runs quite well where the virtual red border shows the parking lot filled by the vehicle identified by the camera. 


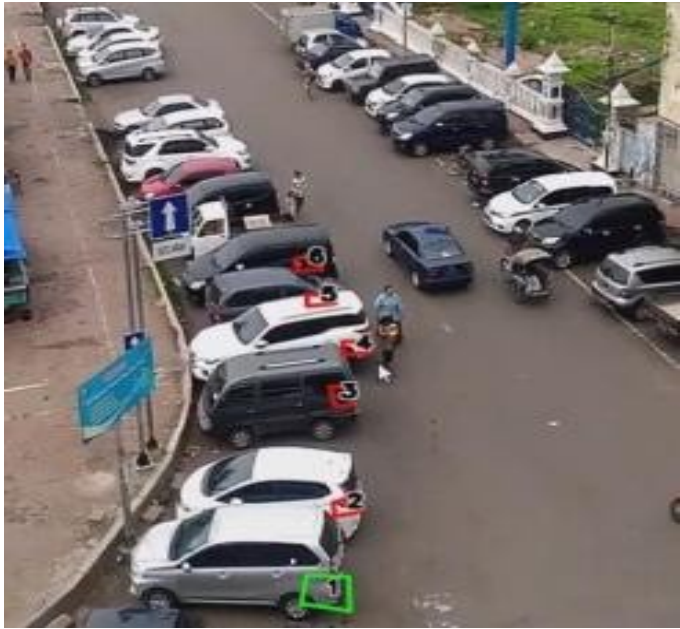

Fig. 7. Results of Parking Vehicle Identification Using Smart Cameras At Day

Fig. 7 shows that the identification of vehicles during the day with different camera angles, the camera capture shows that the camera focuses on only 5 vehicles, one of vehicles object has an error in the identification of the vehicle caused by the position of the virtual border that is not yet fit, so in the running program of errors detecting edges.

The purpose of this testing is to know the speed in sending the real data using modem, the compatibility of the data to be sent and the stability of the communication system. Stages in the testing of the information system is a testing that starts from the data of parking vehicles captured by the camera then the data is sent to the server. Data on the server is processed to be displayed in web-based applications and mobile apps.

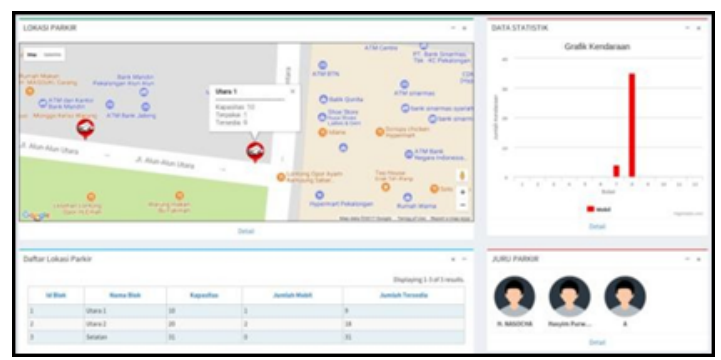

Fig. 8. Display of Desktop Information System

Map based information systems on the desktop with the features provided (in Fig. 8) are as follows:

1) The number of vehicles in the parking area in real time

2) The parking attendant on duty

3) Recapitulation of the number of vehicles per day / week / month / year

While the display in mobile applications are as follows:

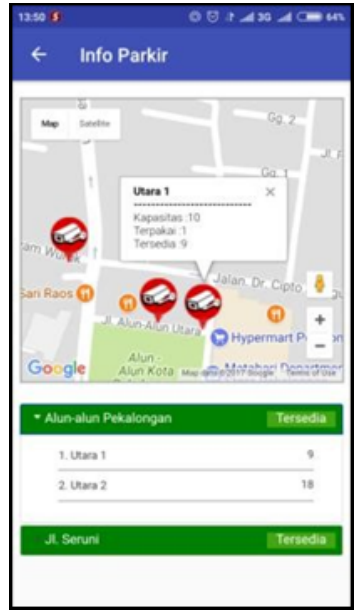

Fig. 9. Display of Mobile Application

The information provided in the mobile application as in Fig. 9. with features as follows:

[1] Amount of parking space available

[2] Location of map-based parking area

\section{CONCLUSSION}

The optimization of parking management will be very useful among others related to the verification between field data manually with real time data, easy in obtaining parking data, ease in preparing daily, weekly, monthly or yearly report as well as easiness in making a policy related to parking.

From the development of integrated parking management system, it is found that the application program related to vehicle identification needs to be improved considering the occasional error in vehicle identification if the vehicle color is the same color as the road color (asphalt).

The initial setting for the camera position becomes an important factor to enter in the virtual boundary area of the parking space.

Prototype of Integrated Parking Management System is an implementation for four-wheeled vehicles or more, so further research is required for the implementation of motorcycle parking.

\section{REFERENCES}

[1] Marcelo RO Castro, Marcio A Teixeira, Luis HV Nakamura, Redolfo I Meneguette "A Prototype of a Car Parking Management Service Based on Wireless Sensor Network for ITS", International Robotics \& Automation Journal, Vol. 2, Issue 3, May 2017.

[2] Annie Sujitg, Chitra S Nair, Manjusha Kulkarni, Shimi Jeyaseelan " A Smart Parking System for A Smart City", IJERCSE, Vol. 3, Issue 6, June 2016.

[3] Faiz Ibrahim Shaikh, Pratik Nirnay Jadhav, Saideep Pradeep Bandarkar, Omkar Pradip Kulkarni, Nikhilkumar B Shardoor, " Smart Parking System Based on Embedded System and Sensor Network", 
International Journal of Computer Application, Vol. 140, No. 12, April 2016.

[4] M. Rashid, A. Musa, M. Ataur Rahman, and N. Farahana, A. Farhana, "Automatic Parking Management System and Parking Fee Collection Based on Number Plate Recognition", International Journal of Machine Learning and Computing, Vol. 2, No. 2, April 2012

[5] Goutham J., Charita B.R, "Cloud-Based Cost Efficient Smart Parking System Based On Iot Technology", International Journal of Advances In Computer Science and Cloud Computing, Volume-5, May.-2017

[6] Patil Vaishali, Pingalkar Nishigandha, Najiya Inamdar, Sonawani Dhawal, Prof.Vijay Sonawane, "A Survey on Smart Car-Parking System Using On Internet-ofThings", International Journal of Innovative Research in Computer and Communication Engineering, Vol. 4, September 2016

[7] Mohammed Raheel Ahmed 1, T C Jermin Jeaunita, "IoT Based Cost Efficient Smart e-Parking System", International Journal of Advanced Research in Electrical, Electronics and Instrumentation Engineering, Vol. 5, November 2016

[8] Ms.Sayanti Banerjee, Ms.Pallavi Choudekar, Prof.M.K.Muju, "Implementation Of Image Processing In Real Time Car Parking System", Indian Journal of Computer Science and Engineering (IJCSE), Vol. 2 No. 115

[9] Ndayambaje Moses , Y. D. Chincholkar, "Smart Parking System for Monitoring Vacant Parking", International Journal of Advanced Research in Computer and Communication Engineering, Vol. 5, June 2016.

[10] Samta Gupta, Susmita Ghosh Mazumdar, "Sobel Edge Detection Algorithm", International Journal of Computer Science and Management Research, Vol 2 Issue 2 February 2013 